\title{
Hydrocarbons emitted by waggle-dancing honey bees stimulate colony foraging activity by causing experienced foragers to exploit known food sources
}

\author{
David C. Gilley, Jacqueline M. Kuzora, Corinna Thom \\ Department of Biology, William Paterson University of New Jersey, Wayne, NJ 07470, USA \\ Received 7 February 2011 - Revised 15 June 2011 - Accepted 7 July 2011
}

\begin{abstract}
Two hypotheses concerning the function of the hydrocarbons emitted by waggle-dancing bees are investigated in this study. First, we test the hypothesis that the waggle-dance compounds stimulate foraging behavior. In support of this hypothesis, the number of bee departures from a hive increased as much as $46 \%$ and the number of forager visits to a feeder station increased as much as $106 \%$ when the dance compounds were introduced into the hive. Second, we test the hypothesis that the waggle-dance compounds stimulate foraging by causing experienced foragers to depart the hive to exploit known food sources. In support of this hypothesis, individually marked foragers visited an empty feeder station both more often and in greater numbers following exposure to the waggle-dance compounds than following exposure to a control substance. The results of these experiments suggest that the waggle-dance compounds function as a foraging semiochemical, operating in synergy with the waggle dance itself.
\end{abstract}

waggle dance / cuticular hydrocarbon / nectar foraging / semiochemical / pheromone

\section{INTRODUCTION}

The waggle dance is a pattern of movement performed by successful honey bee foragers within the nest to recruit colony mates to forage on a profitable food source. This honey bee "dance language", discovered by the pioneering work of Karl von Frisch (von Frisch 1967), is drawing renewed scientific interest as new tools allow novel insights into the remarkable sophistication of the signal, its mechanism of information transfer, and the effect of the signal's message on the dynamic organization of honey bee foraging (see reviews by Dyer 2002; Grüter and Farina 2009). Recently, solid-phase microextraction (SPME) coupled with gas chroma-

Corresponding author: D.C. Gilley,

gilleyd@wpunj.edu

Manuscript editor: Stan Schneider tography and mass spectrometry (GC/MS) analysis added an entirely new facet to the waggle dance signal by revealing a suite of four semi-volatile hydrocarbons (Z-(9)-tricosene, tricosane, Z-(9)-pentacosene, and pentacosane) emitted by waggle-dancing bees (Thom et al. 2007). When a synthetic mixture of this suite of volatile compounds was released into the hives of foraging colonies, it caused bees to exit the hive, suggesting that this suite of volatile compounds stimulates a colony's foraging efforts (Thom et al. 2007). It would not be surprising for a semiochemical to play a role in organizing foraging within honey bee colonies. Olfactory communication is important in the foraging of other social insects (e.g., Free and Williams 1983; Kerr 1994; Vander Meer and Alonso 1998; Steinmetz et al. 2003; Stout et al. 1998) and in organizing nonforaging tasks within honey bee colonies (Free 1987). Determining the role of the waggle-dance 
compounds in the foraging behavior of the honey bee, agriculture's most important pollinator, should be considered a priority.

Here we seek to further our understanding of the waggle-dance compounds beyond the single published account of their discovery (Thom et al. 2007). First, not revealed by experiments to date, is whether the bees that exit the hive in response to the waggle-dance compounds are foragers. Second, if these bees are foragers, then do they subsequently engage in foraging behavior following their departure? If the hydrocarbons emitted by waggle-dancing bees play a role in foraging communication, then their application to the hive must be shown to affect a colony's foraging activity. The first part of the present study tests this hypothesis by exposing bees to the waggle-dance compounds and then measuring the number of visits by bees to a sucrose feeder to which they had been trained.

The second part of the present study investigates the mechanism by which the waggledance compounds might stimulate foraging. Recruitment pheromones such as ant trail pheromones and bumblebee footprint pheromones often function outside the nest (Vander Meer and Alonso 1998; Stout et al. 1998). The results of the experiments by Thom et al. (2007), however, suggest that the waggledance compounds may have behavioral effects within the nest, similar to the recruitment pheromone of bumblebees (Dornhaus and Chittka 2001; Dornhaus et al. 2003; Granero et al. 2005). The waggle-dance compounds may function within the nest to alter the behavior of any or all of the many behavioral-task groups associated with foraging activities. Preliminary data gathered by the authors in 2008 (not presented here) suggested that one task group that might respond to the waggle-dance compounds is the pool of bees that have previously foraged but are currently inactive, hereafter referred to as unemployed experienced foragers following the terminology of Biesmeijer and de Vries (2001). The second part of the present study tests the hypothesis that the waggle-dance compounds stimulate foraging by reactivating unemployed experienced foragers to exploit known food sources. We predicted that foragers which have been trained to a sugar-water feeder and marked to allow for individual identification will increase their foraging activity following introduction of the waggle-dance compounds even when the feeder is empty. Emptying the feeder will eliminate recruitment to it, so that any arriving bees must be experienced foragers familiar with the food source. Also investigated is whether the predicted dance-compound induced boost in visits to the food source can be accounted for by increases in the number of reactivated foragers, or by increases in the foraging rate of reactivated foragers, or both.

\section{MATERIALS AND METHODS}

\subsection{Experiment 1}

Experiments were performed during July and August, 2008, using one colony of approximately 7,500 workers of the Carniolan variety (Apis mellifera carnica) housed in a five-frame hive in an uncultivated field in North Haledon, New Jersey. Foragers were trained to a feeder containing unscented sugar water located approximately $100 \mathrm{~m}$ from the hive. Training consisted of attracting to the feeder foragers at the entrance of the hive, then gradually moving the feeder to the final location (von Frisch 1967). Care was taken to eliminate foragers from foreign colonies by marking the initial recruits with a dot of paint and then subsequently killing any unmarked bees that visited the feeder.

Upon completion of training, two replicates were conducted each day, one in the morning from 09:30 to $10: 30$, and one in the late afternoon from 16:00 to 17:00. Immediately before each experimental replicate, and only at this time, the feeder was filled with sugar solution. To account for fluctuations in the favorability of foraging conditions, the concentration of the sugar solution was adjusted between $5 \%$ and $11 \%$ to prevent overcrowding of the feeder dish (7$\mathrm{cm}$ diameter) by recruited bees. Each replicate began when the first bee arrived at the feeder station, which typically occurred within 5 min of filling the dish at the feeder station. Thereafter, we counted for $40 \mathrm{~min}$ the number of bee visits to the feeder station for the 
duration of every other minute (a total of 20 counts per trial). A visit was defined as a bee landing on the dish and extending her proboscis to the feeder reservoir. Following their visit, the majority of bees flew directly toward the hive after a single looping flight. However, because bees were not individually identifiable in this experiment, it is possible that some bees were counted more than once within a particular 60 -s count. Over counting of visits was minimized by watching the flight of each bee departing from the feeder and excluding any visits by bees returning to the feeder immediately after departure. Another observer simultaneously counted the number of worker exits from the hive for the duration of every other minute. At minute 10, either $300 \mu \mathrm{l}$ of waggledance compound mixture or $300 \mu$ l of pure ethyl ether was introduced into the hive. The waggle-dance compound mixture contained Z-(9)-tricosene, tricosane, and pentacosane, each diluted 1:100 in ether and mixed at a ratio of $1: 2: 3$, respectively (this ratio produced chromatograms with peak heights that approximately matched those from samples of waggle dancers; Thom et al. 2007), and then further diluted 1:10 in ether. The substance was introduced into the hive by pipetting an aliquot of $100 \mu$ onto each of three watch glasses (6-cm diameter) attached to a wooden strip, which was then immediately inserted into the entrance of the hive, effectively centering the three dishes on the bottom of the hive. The dishes remained in the hive until the experimental replicate was complete. To avoid contamination of the solvent dish, a separate but identical set of introduction apparatus was used for the ether and waggle-dance compound introductions. To control for presentation order and time-of-day effects, the order of the substance introduced (waggle-dance compounds or solvent) was alternated each day. To prevent observer bias, the identity of the substance introduced in each replicate was not revealed to the experimenter recording bee visits to the feeder station. The identity of the substance introduced in each replicate was known, however, by the experimenter recording hive departures because this individual also performed the substance introductions. A total of 15 solvent and 14 waggle-dance compound replicates were performed.

To determine whether exposure to waggle-dance compounds increased the number of hive departures, the mean number of bee departures for solvent and waggle-dance compound replicates was compared using a two-sample $T$ test. A similar comparison was made for bee arrivals at the feeder. Before each of these comparisons was made, the data was adjusted in three ways. First, to account for daily fluctuations in foraging conditions, the data for each replicate were normalized by dividing each 1-min count by the average number of hive departures per minute during the 10 min immediately preceding the experiment (for hive departure data) or by dividing each 1-min count by the number of feeder arrivals during the minute immediately preceding substance exposure (for feeder arrival data; the average was not used here because there was a predictable increase in bees arriving from minute 1 to minute 9). Second, all data were natural-log transformed to yield normally distributed sample groups with equal variances. Third, data from morning and afternoon trials were pooled because time of day did not impact behavior at the feeder; the mean bee count just before substance exposure was almost identical between morning ( 8.5 bees $/ \mathrm{min}, 95 \% \mathrm{CI} \pm 2.7)$ and afternoon ( 8.9 bees $/ \mathrm{min}, 95 \% \mathrm{CI} \pm 2.8$ ) replicates.

\subsection{Experiment 2}

Experiments were conducted between June 25 and August 18, 2009, using two colonies of approximately 7,500 workers of the Italian variety (Apis mellifera ligustica) housed in a five-frame hive in an uncultivated field in North Haledon, New Jersey. Foragers were trained, as described for Section 2.1, to a feeder containing unscented sugar water located approximately $100 \mathrm{~m}$ from the hive. Following training of the bees to the feeder, but before experimental replicates began, we marked each bee visiting the feeder with a unique color combination to allow individual identification of the foragers experienced with the feeder-dish food source. Thereafter, the feeder was filled only once each day so that the bees remain trained to it, but this was done at a random time of day to prevent the bees from learning to visit the feeder at a particular time. During each daily feeding, which lasted approximately $60 \mathrm{~min}$, additional foragers were marked to ensure that the majority of visitors to the feeder during each experimental replicate were marked for individual 
identification. The total number of marked bees by the end of the experiment was approximately 84 for colony 1 and 92 for colony 2 .

Each replicate of this experiment consisted of recording for $60 \mathrm{~min}$ the time and identity ("unknown" for unmarked bees, never more than $10 \%$ of the visiting bees) of each forager visiting the empty feeder dish. A visit was defined as a bee landing on the feeder dish, no matter how briefly. Any repeat visits by a bee within $60 \mathrm{~s}$ of the bee's first visit were not considered visits. Introduced into the hive at minute 30 was $300 \mu$ of either waggle-dance compound mixture or this mixture's solvent, ethyl ether. Introduction procedures and composition of the waggle-dance compound mixture were identical to those described for Section 2.1 Also recorded was the number of departures of bees from the hive, which was sampled for $60 \mathrm{~s}$ every fourth minute. Each replicate of the experiment was conducted at one of the following times of day: 09:00, 11:00, 13:00, $15: 00$, or 17:00, determined randomly. Because the feeder must remain empty during data collection, the daily feeding was scheduled so as to not coincide with the experiment. A single replicate was performed each day to insure that each substance exposure was independent of previous exposures. The type of replicate (solvent control or waggle-dance compounds) was determined by coin toss each day, and to avoid observer bias, this information was withheld from the observer at the feeder. Two colonies were used for the experiment. Only a single colony was set up at any one time to prevent competition and drifting among colonies that might obfuscate results. Eleven replicates (six waggle-dance compound and five solvent control introductions) were conducted with colony 1 , and another 11 replicates (five waggle-dance compound and six solvent control introductions) were conducted with colony 2 .

The data generated by the experiment described above yielded three variables upon which we focused our analysis.

1. Number of Visits to Feeder: the total number of bee arrivals at the feeder, including unmarked bees ( $n=11$ replicates for colony $1, n=11$ replicates for colony 2)

2. Number of Visitors to Feeder: the number of unique visitors (i.e., different individuals) that arrived at the feeder, excluding unmarked bees ( $n=11$ replicates for colony $1, n=11$ replicates for colony 2)

3. Number of Feeder Visits per Bee: the number of visits to the feeder by each individual bee, including only those marked bees that participated in at least one dance-compound replicate and one solvent-control replicate $(n=34$ bees for colony $1, n=42$ bees for colony 2 )

To investigate the effects of the dance compounds on each of these three variables, we calculated the difference between the Number of Visits/Number of Visitors/Number of Visits per Bee for the $30 \mathrm{~min}$ before substance introduction compared to the $30 \mathrm{~min}$ after substance introduction. The general linear model was used to determine whether replicate type (solvent control or dance compounds) and colony identity (colony 1 or colony 2) were significant factors with respect to the first two variables (Number of Visits and Number of Visitors). The differences used in the general linear model are expressed as percent changes in Figure 2a, b for ease of comparison. Because the data for the third variable (Number of Feeder Visits per Bee) were self-paired, a repeated measures ANOVA was used to determine the effect of replicate type and colony identity. The repeated measures ANOVA used as subjects the 76 individual bees which visited during both solvent control and dance compound replicates. In cases where an individual bee participated in more than one replicate of either type, we calculated the average difference between visits before and after exposure and used this average in the $T$ test. All three variables were normally distributed with equal variances between both factors.

\section{RESULTS}

\subsection{Experiment 1: number of bee departures from hive}

The average number of departures from the hive following substance exposure depended on whether waggle-dance compounds or solvent was introduced (Figure 1a). The time course of this effect can be divided into three distinct phases: before substance exposure (minutes 19), immediately following substance exposure 
a

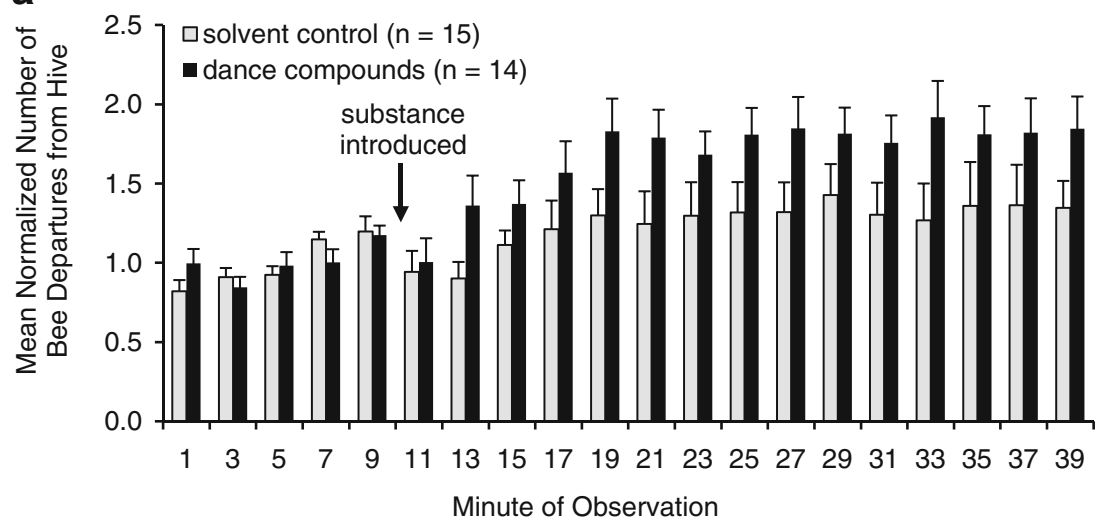

b

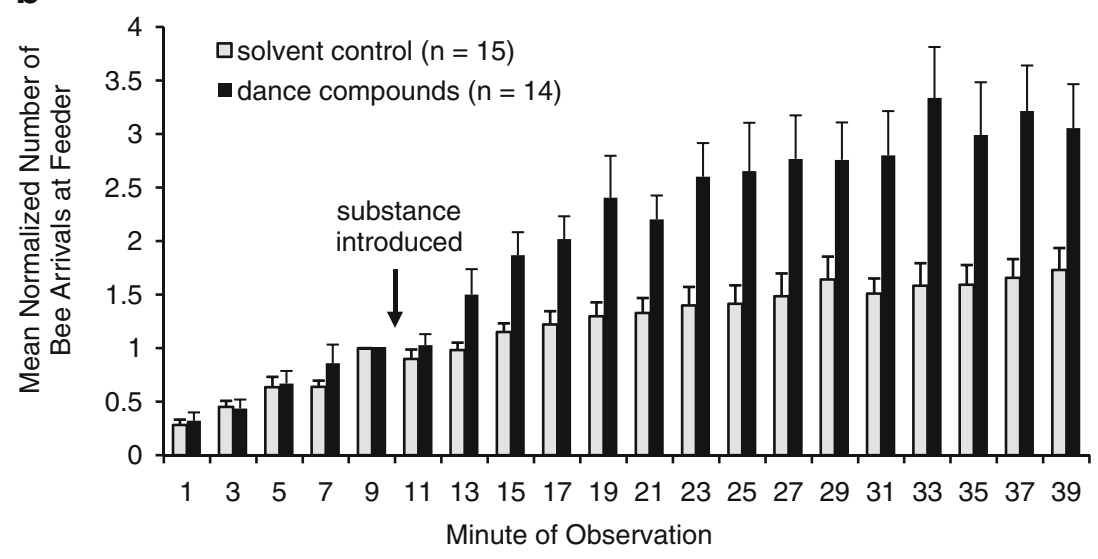

Figure 1. Mean normalized number of bee departures from the hive and arrivals at a feeder station for two treatments over the course of 29 40-min trials. At minute 10, either the waggle-dance compound mixture or the mixture's solvent, ether, was introduced into the hive. Error bars indicate one standard error. a Bee departures from the hive. Counts for each replicate were normalized to the average number of bees departing between minutes 1 and 9. b Bee arrivals at feeder station. Counts for each replicate were normalized to the number of bees arriving at minute 9 .

(minutes 11-19), and the second half of the experiment (minutes 21-39). Before substance exposure, there was no difference in departures between waggle-dance compound and control exposures (two sample $T$ test of average number of departures between minutes 1 and $9-T$ value $=1.47, P$ value $=0.153, d f=27$ ). Immediately following substance exposure, there was a momentary decrease in the number of departures, which was more quickly recovered from during waggle-dance compound than control replicates (Figure 1a, minute 13), though the overall trend for this period of the experiment did not significantly favor waggle-dance over control exposures (two sample $T$ test, minutes $13-19-T$ value $=-1.74, P$ value $=0.094, d f=27$; minute 11 removed from analysis to avoid artifacts of the disturbance resulting from introduction of the compounds into the hive). During the last half of the experiment, there was little change in the average number of departures, but there were significantly more depar- 
tures during waggle-dance compound exposures than during control exposures (two sample $T$ test, minutes $21-39-T$ value $=-2.11, P$ value $=$ $0.044, d f=27)$.

\subsection{Experiment 1: number of bee arrivals at feeder}

The average number of arrivals at the feeder following substance exposure depended greatly upon whether waggle-dance compounds or solvent was introduced (Figure 1b). The time course of this effect can be divided into the same three phases as above: before substance introduction (minutes 1-9), immediately following substance introduction (minutes 11-19), and the second half of the experiment (minutes 2139). Before substance introduction, there was again no difference in arrivals between waggledance compound and control exposures (two sample $T$ test of the average number of arrivals between minutes 1 and $9-T$ value $=-0.52, P$ value $=0.610, d f=27)$. Immediately following substance introduction at minute 10 , feeder arrivals remained unchanged (Figure $1 b$, minute 11 ), but then increased significantly more quickly during waggle-dance compound than during control exposures (two sample $T$ test, minutes 11 to $19-T$ value $=-3.80, P$ value $\leq$ $0.001, d f=27)$. During the last half of the experiment, feeder arrivals increased slowly for both types of substance introductions, but by this point arrivals during waggle-dance compound exposures outnumbered those during control exposures by nearly a factor of two (two sample $T$ test, minutes 21 to $39-T$ value $=$ $-3.61, P$ value $\leq 0.001, d f=27$ ). This difference between waggle-dance compounds and control exposures remained through to the end of the experiment, $30 \mathrm{~min}$ after substance introduction (Figure 1b, minute 39).

\subsection{Experiment 2: number of visits to feeder}

The number of visits to the empty feeder dish was significantly greater following exposure to the waggle-dance compounds compared to the solvent control (general linear model, $F=14.69$, $P \leq 0.001, d f=1,19)$, while colony was not a significant factor affecting the number of visits $(F=0.02, P=0.895, d f=1,19)$. Data from the two colonies are therefore pooled into one data set, and this is displayed as percentage changes in Figure 2a.

\subsection{Experiment 2: number of visitors to feeder}

The number of unique visitors to the empty feeder was significantly greater following exposure to the waggle-dance compounds compared to the solvent control (general linear model, $F=$ 6.42, $P=0.020, d f=1,19$ ), while colony was not a significant factor affecting the number of visits $(F=0.28, P=0.606, d f=1,19)$. Data from the two colonies are therefore pooled into one data set, and this is displayed as percentage changes in Figure $2 b$.

\subsection{Experiment 2: number of feeder visits per bee}

The number of feeder visits per bee was significantly greater following exposure to the waggle-dance compounds compared to the solvent control (repeated measures ANOVA, $F=14.28, \quad P=0.0003, d f=1,75)$ while colony was not a significant factor $(F=2.58, P=$ $0.1107, d f=1,75)$. The mean difference for all 76 bees (from both colonies) between the number of visits before and after substance introduction for solvent control exposures was -0.75 , and for dance-compound exposures was -0.07 . These means are displayed in Figure 2c.

\subsection{Experiment 2: number of bee departures from hive}

The results concerning the number of bee departures from the hive do not directly relate to the hypothesis under investigation in the second part of this study, so they are only summarized here. This summary is included to verify the effect of the dance compounds on hive departures observed in a single colony in Section 2.1. 
a

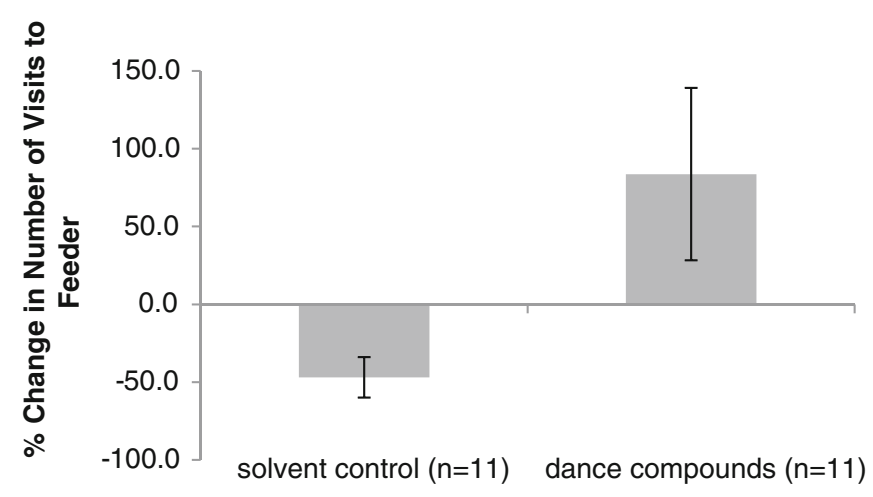

b
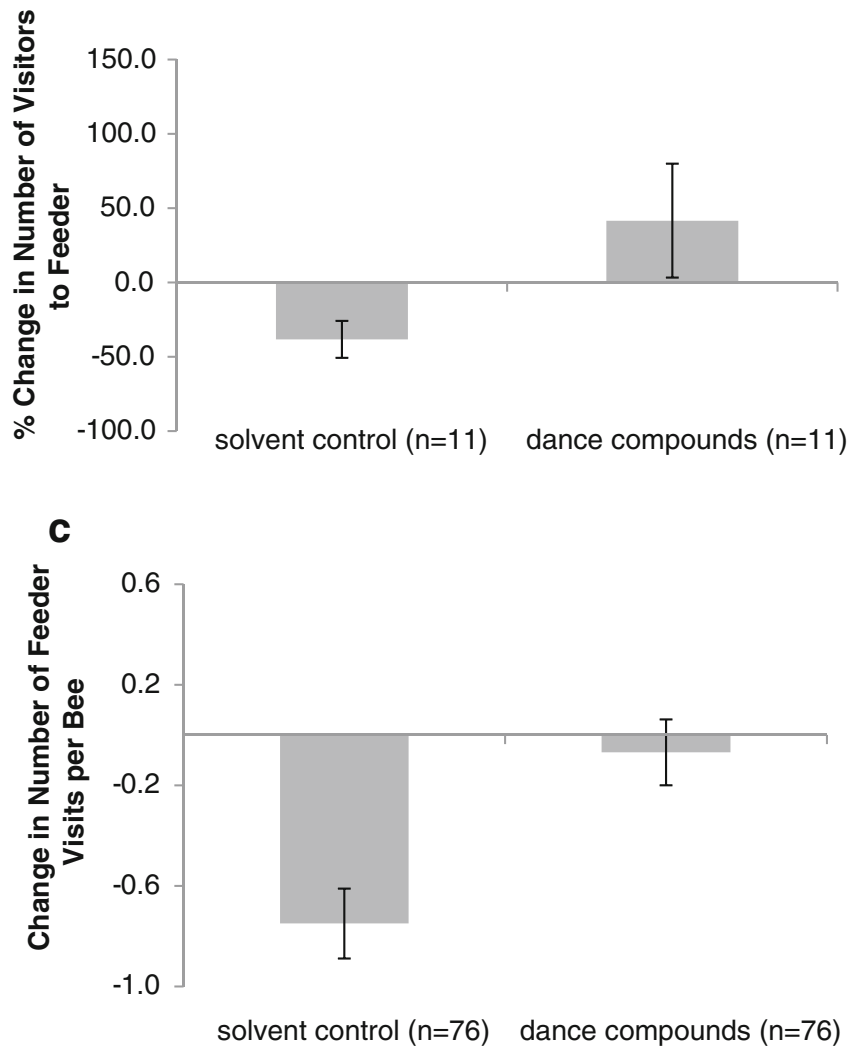

Figure 2. Comparison of changes in foraging behavior following exposure to waggle-dance compounds and a solvent control. Change is the difference between the bees' behavior during the 30 min prior to substance introduction and the $30 \mathrm{~min}$ following substance introduction. Error bars indicate one standard error. a The

Two approaches were taken to assess the bee departures from ten replicates using colony effect of the dance compounds on the number of 1 and 12 replicates using colony 2 were pooled. departures from the hive. First, the number of For each replicate, we tallied the number of 
departures for minutes $30-40$ (i.e., the $10 \mathrm{~min}$ following substance introduction) and the number of departures for minutes 41-60 (i.e., through to the end of the experiment). The percent change was then calculated by comparing these two time intervals to the number of departures during minutes 0-29 (i.e., before substance introduction). The mean percent changes for solvent control replicates was $-43 \%$ and $-22 \%$ for minutes $30-40$ and minutes 41-60, respectively, while the mean percent changes for waggle-dance compound replicates were $-30 \%$ and $+12 \%$ for minutes 30-40 and minutes 41-60, respectively. Second, a general linear model using changes in departures and incorporating replicate type (waggledance compound or control), time interval (minutes $30-40$ or minutes $41-60$ ), and colony (1 or 2) showed both replicate type and time interval as highly significant factors while colony was not $(F=10.54, P=0.002$ for replicate type; $F=21.7, P \leq 0.001$ for time interval; and $F=0.01, P=0.941$ for colony; all $d f=1,40$ ).

\section{DISCUSSION}

The results from the two experiments presented here suggest two major conclusions. First, the hydrocarbons emitted by waggledancing bees affect the behavior of bees by stimulating foraging activity. This was shown in the first experiment by the immediate rise and sustained high level of bee departures from the hive (Figure 1a) and bee arrivals at the feeder (Figure 1b) following exposure to a blend of three waggle-dance compounds. Some of this rise in foraging activity following exposure was no doubt due to natural waggle-dance recruitment to the sucrose solution available at the feeder, but comparison to control exposures shows that bee departures increased over control levels by as much as $46 \%$ and bee arrivals increased by as much as $106 \%$. These increases in foraging activity over control levels must be due to the effect of the waggle-dance compounds. The difference in arrivals between dance compound and solvent control treatments was highly significant for the single colony tested in Section 2.1. Similar results were obtained in Section 2.2 for two additional colonies of a different subspecies during a different year (Figure 2a; shown are comparisons of before and after substance introduction, not the timescale of difference as in Figure 1). The observed responses of bees to the waggledance compounds are therefore not likely idiomatic, suggesting that the waggle-dance compounds play an important role in organizing foraging tasks within honey bee colonies.

The second major conclusion to be drawn from the experiments described in this study is that the hydrocarbons emitted by waggledancing bees increase foraging activity at least partly by means of reactivation, i.e., by stimulating foragers with knowledge of a food site to depart the hive and fly to the location of the food. Following exposure to the waggle-dance compounds, foragers visited an empty feeder in greater numbers (Figure 2b) and more often (Figure 2c) than following exposure to the solvent control. Because the feeder was empty, these increases over control levels cannot possibly be explained by enhanced recruitment to the feeder, but must be due to stimulation of foragers with experience foraging at the feeder. These experienced foragers are not only reactivated by the dance compounds but also stimulated to forage at a faster rate than the bees visiting the feeder during control replicates, which were either inspectors spontaneously checking known food sources or experienced foragers reactivated by a stimulus other than the introduced dance compounds. This increase in foraging rate (i.e., number of visits per bee over $30 \mathrm{~min}$ ) was relative rather than absolute. The absolute change in foraging rate for 76 bees when exposed to the dance compounds was not different from zero (Figure 2c). However, when compared to the control treatment, which contained the same amount of the solvent, the waggle-dance compounds had a significant stimulating effect $(P=$ $0.001)$. The depressant effect of the solvent on foraging behavior, which is seen throughout the data presented here, appears to be working in opposition to the stimulating effect of the dance 
compounds. Temporary disruption of foraging is not a particularly surprising response to ether, but use of such a solvent was unavoidable given the nature of the waggle-dance compounds.

The conclusions from the experiments described in this study imply that the hydrocarbons emitted by waggle-dancing bees are a semiochemical that functions in concert with the information contained in the waggle dance to speedily and efficiently direct foragers to exploit available food sources. While the waggle dance advertises profitable food locations, the compounds emitted by the dancers may advertise to a wider audience favorable foraging conditions. A close comparison might be drawn to the foraging pheromone of bumblebees, which is released by bumblebee foragers upon returning from a successful foraging trip (Dornhaus and Chittka 2001; Dornhaus et al. 2003; Granero et al. 2005) and appears to act as a general foraging activation signal, indicating favorable foraging conditions. This pheromone, a collection of terpenes (Granero et al. 2005), is not chemically related to the long-chain hydrocarbons produced by honey bee waggle dancers. Similarity in function may therefore indicate convergent evolution. Within a honey bee nest, foragers with current memories of profitable food sites would be an important task group receiving and responding to a signal indicating favorable foraging conditions. Stimulation of experienced foragers by the waggle-dance compounds might explain why previous studies have found that experienced foragers can become reactivated after following dances very briefly $(<5$ s) or not at all (Gil and Farina 2002; Biesmeijer and Seeley 2005). However, we do not yet know whether a forager needs to follow a dance to be stimulated by the waggle-dance compounds, so this connection remains tentative. Reception of the waggle-dance semiochemical may also explain why exposure of a non-foraging honey bee colony to the dancefloor odors of a foraging colony increases the number of foragers visiting an empty feeder (Thom and Dornhaus 2007), and why, in response to dancers indicating an unknown food source with an unfamiliar scent, most bees
(82\%) fly to previously visited food sites (Grüter et al. 2008). Floral food-source odors, which are known to reactivate experienced but inactive foragers in much the same way as the waggledance compounds (Reinhard et al. 2004), may be an alternative explanation for the former result (i.e., Thom and Dornhaus 2007), but cannot explain the latter (i.e., Grüter et al. 2008) because the food odor emanating from the dancer did not match the site with which the foragers were familiar. The discovery of the waggle-dance compounds' role in the reactivation of nectar foragers also reinforces Grüter and Farina's (2009) emphases regarding (a) the importance in honey bee foraging of private information about food sources, and (b) the clarification that the waggle-dance language is only one component of the multimodal, multicomponent waggle-dance signal and synonymous use of these terms should be avoided.

\section{ACKNOWLEDGMENTS}

The authors would like to thank Kristina Kupryk, Murat Mamkegh, and Chad Socha for assistance with early versions of experiment 1 , and Krystle Frederick for assistance with experiment 2. Bhanu Chauhan and Stephen Vail provided helpful advice on experimental design and data analysis. For providing and assisting with the apiary and experimental field site, we thank George Arsnow, Dillon Christo, and the LANXESS Corporation. Support for D. Gilley was provided by William Paterson University's Center for Research and Assigned Release Time Program.

Des hydrocarbones émises par des abeilles effectuant la danse frétillante stimulent l'activité de butinage de la colonie en incitant les butineuses expérimentées à exploiter des sources de nourriture connues

Danse frétillante / hydrocarbone cuticulaire / récolte de nectar / substance semiochimique / phéromone

Von schwänzeltanzenden Bienen abgegebene Kohlenwasserstoffe regen erfahrene Sammelbienen dazu an, bekannte Futterquellen auszubeuten und steigern dadurch die Sammeltätigkeit des Volkes

Schwänzeltanz / kutiluläre Kohlenwasserstoffe / Nektarsammeln / Botenstoff / Pheromon 


\section{REFERENCES}

Biesmeijer, J.C., de Vries, H. (2001) Exploration and exploitation of food sources by social insect colonies: a revision of the scout-recruit concept. Behav. Ecol. Sociobiol. 49, 89-99

Biesmeijer, J.C., Seeley, T.D. (2005) The use of waggle dance information by honey bees throughout their foraging careers. Behav. Ecol. Sociobiol. 59, 133-142

Dornhaus, A., Chittka, L. (2001) Food alert in bumblebees (Bombus terrestris): possible mechanisms and evolutionary implications. Behav. Ecol. Sociobiol. 50(6), 570-576

Dornhaus, A., Brockmann, A., Chittka, L. (2003) Bumble bees alert to food with pheromone from tergal gland. J. Comp. Physiol. A 189(1), 47-51

Dyer, F.C. (2002) The biology of the dance language. Annu. Rev. Entomol. 47, 917-949

Free, J.B. (1987) Pheromones of Social Bees. Cornell University Press, Ithaca

Free, J.B., Williams, I.H. (1983) Scent-marking of flowers by honey bees. J. Apic. Res. 22, 86-90

Gil, M., Farina, W.M. (2002) Foraging reactivation in the honeybee Apis mellifera L.: factors affecting the return to known nectar sources. Naturwissenschaften 89, 322-325

Granero, A.M., Sanz, J.M.G., Gonzalez, F.J.E., Vidal, J. L.M., Dornhaus, A., Ghani, J., Serrano, A.N., Chittka, L. (2005) Chemical compounds of the foraging recruitment pheromone in bumblebees. Naturwissenschaften 92, 371-374
Grüter, C., Farina, W.M. (2009) The honeybee waggle dance: can we follow the steps? Trends Ecol. Evol. 24(5), 242-247

Grüter, C., Balbuena, M.S., Farina, W.M. (2008) Informational conflicts created by the waggle dance. Proc. Roy. Soc. Lond. B 275, 1321-1327

Kerr, W.E. (1994) Communication among Melipona workers (Hymenoptera: Apidae). J. Insect Behav. 7(1), 123-128

Reinhard, J., Srinivasan, M.V., Zhang, S. (2004) Scenttriggered navigation in honeybees. Nature 427, 411

Steinmetz, I., Schmolz, E., Ruther, J. (2003) Cuticular lipids as trail pheromone in a social wasp. Proc. Roy. Soc. Lond. B 270, 385-391

Stout, J.C., Goulson, D., Allen, J.A. (1998) Repellent scent-marking of flowers by a guild of foraging bumblebees (Bombus spp.). Behav. Ecol. Sociobiol. 43, 317-326

Thom, C., Dornhaus, A. (2007) Preliminary report on the use of volatile compounds by foraging honey bees in the hive (Hymenoptera: Apidae: Apis mellifera). Entomol. Gen. 29, 299-304

Thom, C., Gilley, D.C., Hooper, J., Esch, H.E. (2007) A scent to the waggle dance. PLoS Biol. 5(9), e228. doi:10.1371/journal.pbio.0050228

Vander Meer, R.K., Alonso, L.E. (1998) Pheromone directed behavior in ants. In: Vander Meer, R.K., Breed, M.D., Winston, M.L., Espelie, K.E. (eds.) Pheromone communication in social insects, pp. 159-192. Westview, Boulder

von Frisch, K. (1967) The Dance Language and Orientation of Bees. Belknap Press of Harvard University Press, Cambridge 